\title{
LA ADMINISTRACIÓN DEL RIESGO CAMBIARIO EN AMBIENTES DE CONTINGENCIA DE LAS EMPRESAS IMPORTADORAS Y EXPORTADORAS DE OAXACA Y CIUDAD DE MÉXICO*
}

\author{
Foreign exchange risk management of import export companies in Oaxaca and Mexico City \\ in contingency environments
}

\author{
Araceli Hernández-Jiménez iD \\ PhD en Ciencias de la Administración, Universidad del Istmo. Oaxaca-México, \\ araceli@bianni.unistmo.edu.mx
}

\begin{abstract}
Cómo referenciar / How to cite
Hernández-Jiménez, A. (2018). La administración del riesgo cambiario en ambientes de contingencia de las empresas importadoras y exportadoras de Oaxaca y Ciudad de México. Revista CEA, 4(7), 13-28. https://doi.org/10.22430/24223182.755
\end{abstract}

Recibido: 17 de julio de 2017

Aceptado: 29 de septiembre de 2017

\section{Resumen}

Las empresas importadoras y exportadoras, en comparación con las que no comercializan al exterior, tienen un riesgo adicional caracterizado por la volatilidad cambiaria. En esta investigación, se analizan las características que distinguen a la administración de las empresas con operaciones internacionales de la ciudad de México y Oaxaca, con altos rendimientos ante el riesgo cambiario 2014-2016 en ambientes contingentes, versus las empresas con bajos rendimientos, donde según la teoría de la contingencia las empresas usan diferentes mecanismos para adaptarse a los cambios del medio ambiente. Se usó una regresión logística (RL) para clasificar a las empresas con rendimientos superiores al $20 \%$, en función a sus características en la administración. Para caracterizar la administración se utilizaron 23 variables explicativas que abarcaron aspectos del administrador y los esquemas de cobertura empleados. Los resultados obtenidos encontraron que solo cuatro de las 23 variables fueron estadísticamente significativas para que las compañías lograran altos márgenes de rentabilidad, sobresaliendo la utilización de instrumentos financieros derivados como un factor determinante.

Palabras clave: riesgo cambiario, instrumentos financieros derivados, teoría de contingencia.

\begin{abstract}
Compared to companies that do not market products abroad, import export businesses face an additional risk: foreign exchange volatility. This work analyzes the features that distinguish the management strategies of highly profitable companies that conduct international businesses in Oaxaca and Mexico City in contingency environments - the 20142016 foreign exchange risk-from low-profit ones. According to the contingency theory, the
\end{abstract}

\footnotetext{
*Este artículo es parte de una investigación más amplia denominada: «La administración del riesgo cambiario en las empresas importadoras y exportadoras del Istmo Oaxaqueño y de la Ciudad de México», financiada por el Programa para el Desarrollo Profesional Docente, oficio de autorización DSA/103.5/16/10253.
} 
former use different mechanisms to adapt to changes in the environment. Logistic Regression (LR) was used to classify companies with profits above $20 \%$ based on their management features. To characterize the management style, 23 explanatory variables were employed; they dealt with aspects related to the administrator and the cover schemes. The results revealed that only 4 , out of those 23, were statistically significant for companies to achieve high profit margins and the use of derivative instruments stood out as a decisive factor.

Keywords: Foreign exchange risk, derivative instruments, contingency theory.

\section{INTRODUCCIÓN}

En las negociaciones comerciales internacionales se tiene un riesgo adicional, en comparación con el comercio nacional, el cual proviene de la incertidumbre conexa con los tipos de cambio (Levi, 2006). La globalización ha dado un mayor auge al comercio internacional y a los mercados financieros. La diversificación de productos financieros y el resurgimiento de la volatilidad a nivel mundial, ha incrementado la importancia del riesgo del tipo de cambio, el cual, ha provenido representado por un aumento en la volatilidad de los tipos de cambio para las empresas internacionales (Levi, 2006).

México es la principal nación exportadora de América Latina y la décimo quinta a nivel global, se ha convertido en un centro de comercio internacional (PROMÉXICO, 2016) (SE, 2016). La proximidad al mercado de exportación de Estados Unidos de América sigue siendo una ventaja competitiva y ha suscrito 12 acuerdos con 46 países (OCDE, 2017). A finales de 2016, las exportaciones totales mostraron un crecimiento anual de 6,6 \%, las dirigidas a Estados Unidos avanzaron
$3,5 \%$, y las canalizadas al resto del mundo lo hicieron en 11,6\% (INEGI, 2017).

Las exportaciones en Oaxaca en 2015 disminuyeron el $45 \%$ respecto al 2014, colocándose entre los siete estados con menos registro de exportaciones (INEGI, 2017). EI $72 \%$ de las exportaciones de Oaxaca se dirige a Estados Unidos, sin embargo, solo representa el $5,7 \%$ del PIB estatal.

El entorno actual de las empresas en México, en relación con el sistema de tipo de cambio, se tiene un régimen de libre flotación, mismo que bajo este esquema ha atravesado situaciones de alta volatilidad cambiaria, en el periodo que comprende de 2014-2016, ha habido depreciaciones abruptas del peso frente al dólar de más del 70 \%. En escenarios contingentes, el administrador debe tomar decisiones, estableciendo coberturas para minimizar el riesgo cambiario. Las decisiones financieras son trascendentales, donde la calidad de las decisiones se verá reflejado en el éxito o fracaso de la empresa (Ross, Westerfield \& Jaffe., 2005).

La administración del riesgo financiero en empresas con negocios internacionales es de vital importancia, porque es un riesgo que afecta a sus activos, pasivos y utilidades, dado que tienen que importar insumos, exportar productos, contratar créditos en moneda extranjera, etc., por lo cual, es necesario que existan instrumentos idóneos que permitan administrar el riesgo financiero al que están expuestas las empresas, particularmente, importadoras y exportadoras.

Los instrumentos derivados se han desarrollado desde hace varias décadas y se les considera una herramienta idónea para administrar el riesgo financiero. El mercado de derivados tiene razón de ser ante cualquier escenario riesgoso. Con los derivados es posible administrar el riesgo en dos sentidos, 
por ejemplo, el riesgo puede ser sustituido por certeza (contratos futuros) o sustituir el riesgo perjudicial y operar solamente el riesgo beneficioso (opciones).

Los sociólogos Burns y Stalker (1961), Lawrence y Lorsch (1967), aportaron la teoría de la contingencia ambiental, donde sostienen que la estructura de una organización debe basarse en las condiciones que enfrenta en su entorno. La globalización coloca a la organización en un escenario distinto que afecta sus objetivos, funciones, decisiones y ventaja competitiva. Las organizaciones con entornos cambiantes, deben adaptarse a cualquier circunstancia. Chandler (1990) señala que el medio ambiente obliga a que las empresas adopten nuevas estrategias. La teoría de la contingencia destaca la eficacia de las acciones administrativas emprendidas en una situación y rechaza de cierta forma la estandarización, así la tecnología, el tamaño y el entorno son factores imprescindibles.

En una organización inestable surgen cambios impredecibles, por consiguiente, se requiere de mayor flexibilidad interna que le permita responder de manera eficaz a los cambios externos. En estas condiciones, habrá mayor delegación de autoridad para la toma de decisiones. La dirección empresarial debe contemplar los escenarios de crisis, dado que repercuten en las operaciones de las empresas. Para Damico (2011), una crisis financiera puede hacer impactar repentinamente el tipo de cambio, el cual es una variable de suma importancia en los negocios, ya que su nivel puede tornar inviable o muy rentable un negocio (López, 2010).

Desde la perspectiva de la teoría de la contingencia, es importante definir las acciones administrativas apropiadas en una situación determinada, dependiendo de los parámetros particulares de la situación. En esta investigación se analizan las características de la administración para enfrentar los diferentes riegos, enfocándose específicamente en los esquemas de cobertura para minimizar el riesgo cambiario que utilizan las empresas de la ciudad de México y las de Oaxaca.

\section{MARCO TEÓRICO-EMPÍRICO}

\section{La gestión del riesgo cambiario en empresas con operaciones internacionales}

Kauffman (2000) ha señalado que la globalización de los mercados coloca a la empresa en un escenario distinto que afecta sus objetivos, funciones, decisiones y ventaja competitiva. Bajo la perspectiva modernista es necesario realizar el análisis del medio ambiente en general y el entorno internacional. Hatch (1997) afirma que las organizaciones interactúan con otros miembros de su entorno. La importancia del ambiente externo de la organización se hizo evidente con la teoría de los sistemas abiertos y la teoría de las contingencias.

Actualmente, los cambios que se están operando en el entorno externo de las organizaciones solicitan que se tenga mayor atención por parte de los que toman decisiones. A través de las problemáticas que están ocurriendo en el ambiente pueden verse afectadas en las organizaciones. El análisis al entorno internacional y la globalización, las organizaciones pueden ser incorporadas dentro de múltiples redes que son influenciados por las condiciones generales y las tendencias en sus propios entornos, así como los entornos de aquellos con quienes interactúan.

La planificación del desempeño económico financiero permite a las empresas internacionales desarrollar estrategias para competir en el mercado global (López, 2010), en donde la calidad de la gestión es un factor 
determinante, en el que los administradores crean, dirigen y operan las organizaciones hacia ciertos propósitos, a través del esfuerzo humano coordinado y cooperativo (Koontz \& Weihrich, 2012; Follet, 1960) y otros recursos según McFarland (1974), donde la administración del riesgo cambiario juega un papel importante en lo que se refiere a los objetivos financieros de las organizaciones.

Una de las tareas principales del administrador es generar valor a la empresa, siendo un elemento clave las características personales de este. Diversos estudios se enfocan en conocer sus características personales, tales como el nivel educativo (Veciana, 1989; Storey, 1994; Cooper et al., 1994; Ucbasaran, et al., 2008; Kong \& Zhang, 2010), género (Lewis, 2006; Chowdhury \& Endres 2005), edad (Entrialgo, 2000; Gupta \& York, 2008; Fernández \& Junquera, 2001), las cuales influyen en el desempeño empresarial (McMillan, et al., 1985) y el logro de ventajas competitivas (Ong, et al., 2010). Según Chell (1991), las características personales pueden inhibir el crecimiento potencial y finalmente amenazar la supervivencia de la empresa.

En lo que se refiere a la importancia de la gestión para lograr los objetivos financieros de las empresas con operaciones internacionales, diversos autores (García \& Avella, 2007; Zapata, 2005; Cadogan et al., 1999; Kaynak \& Kothari, 1984), encuentran una relación directa entre la postura del dirigente de la organización ante el riesgo y la internacionalización de la empresa. Una relación negativa indica que, a mayor aversión al riesgo, menor será la penetración al mercado internacional. Una relación positiva es la aceptación del riesgo conlleva a la internacionalización de la empresa.

Kohli y Jaworski (1993) afirman que la aversión al riesgo afecta negativamente el rendimiento de la organización ya que limita la toma de decisiones para ingresar a nuevos mercados, desarrollar nuevos productos y responder rápidamente a los cambios del entorno.

Sierra y Londoño (2010) a través de su investigación realizada a 200 empresas manufactureras en Colombia sobre el uso de derivados financieros, encontraron que su uso es importante en la gestión del riesgo, dado que influye directamente en el patrimonio de las organizaciones y en su potencial de generación de valor y además señalan que las empresas que más usan derivados son aquellas que están expuestas mediante algún canal internacional, especialmente las que importan y exportan o que buscan financiación en el extranjero.

Jorion (1990) encontró que el nivel de cobertura guardaba una relación directa con la proporción de ventas en el extranjero. Dolde (1993) en su investigación encontró evidencia de que más del $45 \%$ de las empresas estadounidenses de su muestra tenían un profesional a tiempo completo dedicado a la gestión del riesgo, y al menos el $15 \%$, tres o más. Adicionalmente, más del 20\% habían implantado centrales de gestión a través de complejos sistemas informáticos.

Una de las tareas del administrador, es la gestión del riesgo, en donde se analiza el tipo de cobertura que se empleará, resaltando la cobertura natural, caracterizada en las empresas con ingresos en moneda extranjera que pueden endeudarse en esa moneda para efectuar una compensación entre los flujos de activo y pasivo y, de esta manera, reducir o eliminar el riesgo cambiario (Kedia \& Mozumdar, 2003).

\section{Cobertura cambiaria}

Las compañías están expuestas a diversos riesgos por la volatilidad cambiaria y que según Madura (2009), se clasifican en: 
Exposición por transacciones de efectivo futuras ante las variaciones cambiarias, derivadas por las posiciones en cuentas por pagar y por cobrar futuras en varias divisas.

Exposición económica u operativa, es cualquier exposición de flujos de efectivo (directos o indirectos) a las variaciones cambiarias. Estos provienen de los gastos que se producen por el funcionamiento de las empresas.

Exposición a la conversión, es la exposición de los estados financieros consolidados de una empresa a las variaciones cambiarias. Medirla les permite a las empresas pronosticar en cada utilidad $y$, después determinar cómo dichas utilidades pueden ser afectadas por potenciales variaciones en los tipos de cambio de cada divisa.

Una estrategia de cobertura consiste en emplear instrumentos financieros que contrarresten de manera eficiente las pérdidas o ganancias que la empresa sufre en su posición primaria debidas a los movimientos en los niveles de las diferentes variables (Arango \& Arroyave, 2011).

El mercado de derivados ofrece diversos instrumentos que pueden utilizarse con fines de cobertura o especulación, en México funciona el Mercado Mexicano de Derivados, conocido como Mexder, y resulta interesante investigar si las pymes mexicanas utilizan estos instrumentos. Un derivado es un instrumento financiero, el cual está vinculado al valor de un activo subyacente, es un contrato que deriva del precio futuro para tal activo sobre la base de su precio actual y las tasas de interés (Gray \& Place, 2003). El subyacente puede ser un activo físico o un activo financiero como una divisa, tasa de interés, acción, entre otros.

La utilización de instrumentos derivados es una decisión del administrador de la empresa quién adoptará decisiones en cuanto a los niveles de tipo de cambio, y con ello decidirá las estrategias a utilizar para realizar las coberturas. El objetivo de la cobertura con instrumentos derivados no es especular con la variabilidad del activo subyacente, sino asegurar una rentabilidad fijando sus costos o ingresos a un valor previamente determinado (Clavijo \& Roberto, 2009).

\section{METODOLOGÍA DE LA INVESTIGACIÓN}

En esta investigación se analizan las características de la administración para enfrentar los diferentes riesgos, enfocándose específicamente en los esquemas de cobertura para minimizar los efectos del riesgo cambiario que enfrentan las empresas objeto de estudio. Se realiza un estudio comparativo entre las empresas de la Ciudad de México y de Oaxaca, por tener el primer y penúltimo lugar respectivamente de índice de competitividad.

\section{Planteamiento del problema}

Los riesgos de inestabilidad y de crisis financieras son evidentes y aparecen en forma repetitiva en todos los ámbitos económicos, principalmente por los efectos en las fluctuaciones de los tipos de cambio, provocando inestabilidad cambiaria y afectando los flujos comerciales. Damico (2011), señala que el tipo de cambio representa una variable de suma importancia en los negocios, ya que su nivel puede tornar inviable o por el contrario muy rentable un negocio según López (2010).

La volatilidad del tipo de cambio es uno de los riesgos de mercado que afecta en mayor medida a empresas exportadoras e importadoras, por las constantes negociaciones al exterior. Según Levi (2006) existen varias razones por las cuales las coberturas cambiarias deberían ser adoptadas por la empresa, como la existencia de impuestos corporativos de tipo progresivo, 
economías de escala en la cobertura de transacciones, beneficios de comercialización y de empleo, una menor cuantía en los costos de quiebra esperados y una mejor información interna, por mencionar algunas justificaciones, una razón fundamental es la supervivencia de las empresas, pues si una compañía quiebra tiene efectos nocivos para la población y la economía donde desarrolla sus operaciones, específicamente en los índices de desempleo para población y pérdida de impuestos para los gobiernos.

El peso mexicano ha tenido una depreciación alrededor de 50\%, durante el año 2013 se encontraba en niveles de 12 unidades por cada dólar comparado con el primer trimestre de 2017 que se ubica en 19 unidades por dólar. Y por otra parte no existen estudios hasta el momento que evalúen las características de la administración, frente los efectos de la crisis cambiaria de 2014-2017.

En México, las empresas que realizan operaciones internacionales enfrentan los efectos de la crisis cambiaria 2014-2016, donde los administradores deben tomar decisiones e implementar acciones frente al riesgo cambiario, lo cual constituye un elemento fundamental para que las empresas puedan sobrevivir y obtener los niveles mínimos de rentabilidad exigidos por los empresarios, en ese sentido se plantea la siguiente pregunta de investigación:

¿Qué características distinguen a la administración de las empresas con operaciones internacionales y altos rendimientos ante el riesgo cambiario en un ambiente contingente versus las empresas con rendimientos bajos?

\section{Hipótesis}

Para responder la pregunta de investigación se plantean las siguientes hipótesis:
$\mathrm{H}_{1}$ «Las características personales (edad, nivel de estudios, etc.) de los administradores en la gestión del riesgo cambiario, caracterizan a las empresas con niveles de rendimiento superiores al $20 \%$ versus las empresas con rendimientos menores».

$\mathrm{H}_{0}$ «Las características personales (edad, nivel de estudios, etc.) de los administradores en la gestión del riesgo cambiario, no caracterizan a las empresas con niveles de rendimiento superiores al $20 \%$ versus las empresas con rendimientos menores».

\section{Objetivo de la investigación}

Conocer las características que diferencian a la administración de las empresas con operaciones internacionales y altos rendimientos ante el riesgo cambiario en un ambiente contingente versus las empresas con rendimientos bajos.

\section{Operacionalización}

Las características de la administración: se sitúan el plano del quehacer del administrador, y según Chiavenato (2006), asume el papel de liderazgo, asume riesgos, toma medidas correctivas en disputas o crisis, guía al grupo en las crisis y orienta en los cambios, es en ese sentido que las características de la administración están representadas por una parte por las características del administrador y la otra su quehacer para sufragar los efectos de la crisis en las compañías, que comprende todos aquellos mecanismos que le permiten mantener los niveles de rentabilidad pese a los efectos negativos de los vaivenes cambiarios (Kohli \& Jaworski, 1993), (Kedia \& Mozumdar, 2003), entre los cuales se encuentra parte de los instrumentos financieros derivados. 


\section{Métodos y materiales}

El análisis discriminante es apropiado cuando la variable dependiente es no métrica. Sin embargo, cuando la variable dependiente tiene solo dos grupos, puede preferirse la regresión logística por varios motivos. En primer lugar, el análisis discriminante descansa sobre cumplimiento estricto de los supuestos de normalidad multivariante y la igualdad de matrices de varianzas-covarianzas entre los grupos, supuestos que no siempre se verifican. La regresión logística no se enfrenta a esos supuestos tan estrictos, y es mucho más robusta cuando estos supuestos no se cumplen (Hair, Anderson, Tatham \& Black, 1999, p. 280).

Para esta investigación se usó una regresión logística (RL) que permitió clasificar a las empresas con rendimientos superiores al $20 \%$ conforme a sus características en la administración. La RL permite calcular la probabilidad de ocurrencia de un suceso, en este caso, el que las empresas pertenezcan al grupo de las que obtuvieron rendimientos superiores al $20 \%$, o al otro grupo con rendimiento inferiores, mediante una función de ciertas variables que se presumen relevantes o influyentes, en este caso las variables que describen las características de las administración, donde la variable «Y» es la dependiente y las "X» variables explicativas (independientes y de control), se designan por $X_{1}, X_{2}, \ldots X_{k}$. La ecuación general o función logística es:

$\mathrm{P}(\mathrm{Y}=1)=\frac{1}{1+\exp \left(-\alpha-\beta_{1} X_{1}-\beta_{2} X_{2}-\beta_{3} X_{3}-\ldots \beta_{k} X_{K}\right)}$

Donde:

$\alpha, \beta_{1}, \beta_{2}, \beta_{3 \ldots}, \beta_{k}$ son los coeficientes logísticos correspondientes a cada variable predictora, un coeficiente positivo aumenta la probabilidad de ocurrencia, y un coeficiente negativo la disminuye.

\section{Variables utilizadas}

En la Tabla 1 se presentan las variables utilizadas para el modelo.

Tabla 1. Variables dependientes e independientes

Table 1. Dependent and independent variables

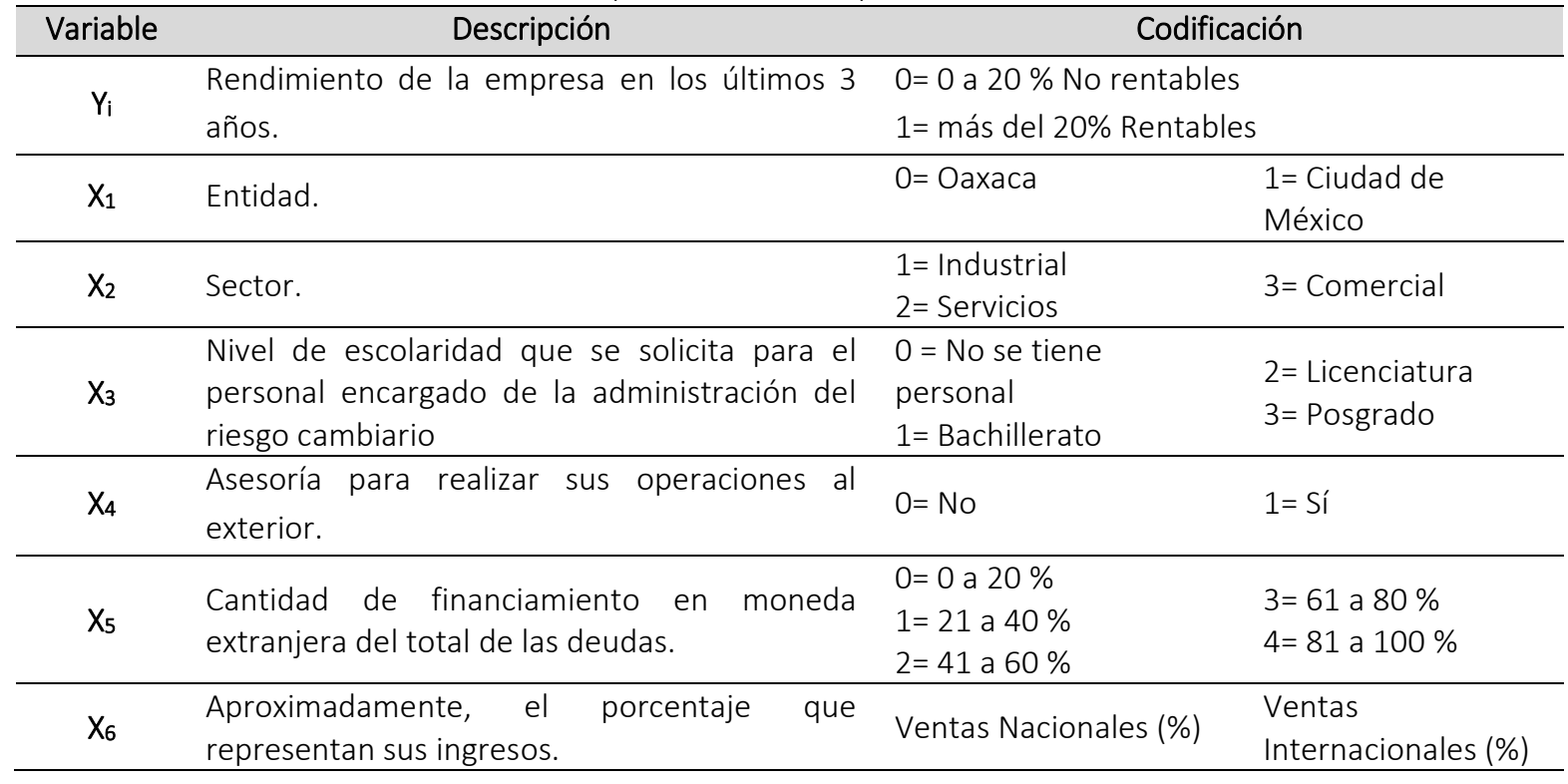




\begin{tabular}{|c|c|c|c|}
\hline$x_{7}$ & $\begin{array}{l}\text { La empresa analiza el riesgo de las variaciones } \\
\text { de tipo de cambio. }\end{array}$ & $0=\mathrm{No}$ & 1= Sí \\
\hline$X_{8}$ & $\begin{array}{l}\text { Rubro que más ha afectado las variaciones de } \\
\text { tipo de cambio. }\end{array}$ & $\begin{array}{l}0=\text { Ninguno } \\
1=\text { Pasivos } \\
2=\text { Activos }\end{array}$ & $\begin{array}{l}3=\text { Resultado } \\
4=\text { Varios }\end{array}$ \\
\hline$X_{9}$ & $\begin{array}{l}\text { Utilización de instrumentos o estrategias de } \\
\text { cobertura para los ingresos en divisa extranjera } \\
\text { para evitar los efectos adversos de una posible } \\
\text { baja de precio de la divisa extranjera. }\end{array}$ & $\begin{array}{l}0=\text { No utiliza } \\
1=\text { Futuros } \\
2=\text { Forwards }\end{array}$ & $\begin{array}{l}3=\text { Opciones } \\
4=\text { Swaps } \\
5=\text { Otro }\end{array}$ \\
\hline$X_{10}$ & $\begin{array}{l}\text { Utilización de instrumentos o estrategias de } \\
\text { cobertura para compras/costos para evitar los } \\
\text { efectos adversos de una posible alza de precio } \\
\text { de la divisa extranjera. }\end{array}$ & $\begin{array}{l}0=\text { No utiliza } \\
1=\text { Futuros } \\
2=\text { Forwards }\end{array}$ & $\begin{array}{l}3=\text { Opciones } \\
4=\text { Swaps } \\
5=\text { Otro }\end{array}$ \\
\hline$X_{11}$ & $\begin{array}{l}\text { Utilización de instrumentos o estrategias de } \\
\text { cobertura para deuda para evitar los efectos } \\
\text { adversos de una posible alza de precio de la } \\
\text { divisa extranjera. }\end{array}$ & $\begin{array}{l}0=\text { No utiliza } \\
1=\text { Futuros } \\
2=\text { Forwards }\end{array}$ & $\begin{array}{l}3=\text { Opciones } \\
4=\text { Swaps } \\
5=\text { Otro }\end{array}$ \\
\hline$X_{12}$ & $\begin{array}{l}\text { El instrumento o estrategia utilizada le ha } \\
\text { servido de protección frente a los riesgos } \\
\text { cambiarios del mercado. }\end{array}$ & $0=$ Nunca. & $\begin{array}{l}1=\text { Siempre } \\
2=\text { Regularmente }\end{array}$ \\
\hline$X_{13}$ & $\begin{array}{l}\text { Cambia la estrategia o el uso de los } \\
\text { instrumentos para los distintos escenarios del } \\
\text { precio de la divisa extranjera. }\end{array}$ & $0=$ No. & 1= Sí \\
\hline$X_{14}$ & $\begin{array}{l}\text { Frecuencia utilizada en instrumentos derivados } \\
\text { para cubrir: gastos en moneda local. }\end{array}$ & $0=$ No se utilizan. & $\begin{array}{l}1=\text { Frecuentemente } \\
2=\mathrm{A} \text { veces }\end{array}$ \\
\hline$X_{15}$ & $\begin{array}{l}\text { Frecuencia utilizada en instrumentos derivados } \\
\text { para cubrir: gastos en moneda distinta a la de } \\
\text { sus estados financieros. }\end{array}$ & $0=$ No se utilizan. & $\begin{array}{l}1=\text { Frecuentemente } \\
2=A \text { veces }\end{array}$ \\
\hline$X_{16}$ & $\begin{array}{l}\text { Frecuencia utilizada en instrumentos derivados } \\
\text { para cubrir: ingresos en moneda distinta a la de } \\
\text { sus estados financieros. }\end{array}$ & $0=$ No se utilizan. & $\begin{array}{l}1=\text { Frecuentemente } \\
2=A \text { veces }\end{array}$ \\
\hline$X_{17}$ & $\begin{array}{l}\text { Comportamiento de las utilidades en relación } \\
\text { al instrumento o estrategia que utilizan. }\end{array}$ & $\begin{array}{l}0=\text { NA (No aplica) } \\
1=\text { Han aumentado }\end{array}$ & $\begin{array}{l}2=\text { Han disminuido } \\
3=\text { Se han mantenido }\end{array}$ \\
\hline $\mathrm{X}_{18}$ & $\begin{array}{l}\text { Cuenta con personal calificado para el manejo } \\
\text { de instrumentos derivados en la empresa. }\end{array}$ & $0=$ No hay personal. & $1=$ Sí \\
\hline$X_{19}$ & Es dueño de la empresa. & $0=\mathrm{No}$ & 1= Sí \\
\hline$X_{20}$ & Es administrador de la empresa. & $0=$ No & 1= Sí \\
\hline$X_{21}$ & Edad del dueño o administrador de la empresa. & $\begin{array}{l}1=20 \text { a } 29 \text { años } \\
2=30 \text { a } 39 \text { años }\end{array}$ & $\begin{array}{l}3=40 \text { a } 49 \text { años } \\
4=50 \text { años o más }\end{array}$ \\
\hline$X_{22}$ & $\begin{array}{l}\text { Máximo grado de estudios del dueño o } \\
\text { administrador de la empresa. }\end{array}$ & $\begin{array}{l}\text { 1= Primaria } \\
2=\text { Secundaria } \\
3=\text { Preparatoria }\end{array}$ & $\begin{array}{l}4=\text { Universidad } \\
5=\text { Posgrado }\end{array}$ \\
\hline$X_{23}$ & $\begin{array}{l}\text { Opinión del riesgo en el negocio, por parte del } \\
\text { dueño o administrador de la empresa. }\end{array}$ & $\begin{array}{l}1=\text { Un gran problema } \\
2=\text { Algo a evitar } 0 \\
\text { eliminar }\end{array}$ & $\begin{array}{l}3=\text { Mantener bajo } \\
\text { control } \\
4=\text { Un gran reto } \\
5=\text { Oportunidad de } \\
\text { negocio }\end{array}$ \\
\hline
\end{tabular}

Fuente: elaboración propia. 


\section{Muestra}

Para recabar la información se encuestaron a los propietarios y/o administradores de las empresas exportadoras e importadoras de la Ciudad de México y Oaxaca, donde el tamaño oscila desde pequeñas a medianas principalmente. El marco de muestreo se estructuró con base en información del SIEM (2016), PROMÉXICO (2016), y se complementó con los datos de empresas que no se encuentran registradas en las bases de datos gubernamentales. La determinación del tamaño de muestra para las empresas asentadas en el estado de Oaxaca, se hace con una precisión de más/menos cinco puntos porcentuales alrededor de la estimación puntual de las proporciones $\left(d_{p}=0.05\right)$ y fijando una confiabilidad de $90 \%(1-\alpha=$ $0.9)$. Bajo el muestreo aleatorio simple para proporciones, de acuerdo con Montesinos et al. (2009), la fórmula para determinar el tamaño de muestra para lograr la precisión deseada al estimar la proporción poblacional, es la siguiente:

$$
n=\frac{N\left[Z_{1-\alpha / 2)}\right]^{2} P Q}{N d_{p}^{2}+\left[Z_{1-\alpha / 2)}\right]^{2} P Q}(1)
$$

Donde:

$d_{p}$ es la precisión de estimación del total poblacional que se está dispuesto a aceptar, $P$ es la proporción de interés, $Q=1-P$, y
$Z_{1-\alpha / 2}$ es el cuantil $1-\alpha / 2$ de la distribución normal estándar.

Con la información anterior, los cálculos en la Ecuación 1 indican que el mínimo de encuestas a levantar para la lograr la precisión deseada con una confiabilidad de $90 \%$ es igual a:

$$
n=\frac{123[1.66]^{2}(0.2)(0.8)}{123(0.05)^{2}+[1.66]^{2}(0.2)(0.8)} \approx 72 \text { Encuestas }
$$

El listado final de las empresas exportadoras e importadoras en la Ciudad de México, que integraron la población objetivo consistió en $N=1227$. Para estimar los parámetros se utilizaron técnicas de remuestreo, boostrap, a falta de información mínima disponible que dicta el muestreo aleatorio simple. Utilizando esta técnica la muestra es $n=35$.

\section{RESULTADOS}

El análisis Logístico Binario se desarrolló en el programa SPSS, mediante el método «por pasos hacia adelante» con el criterio de Wald, en el modelo se van añadiendo al mismo variables independientes según su grado de relación con la variable dependiente y su significación estadística. En la Tabla 2 aparecen 107 casos introducidos, que son los seleccionados para el análisis y los casos excluidos, en este caso son cero.

Tabla 2. Resumen del procesamiento de los casos

Table 2. Case selection

\begin{tabular}{l|r|r}
\hline Casos no ponderados $^{\text {a }}$ & N & \multicolumn{1}{|c}{ Porcentaje } \\
\hline Casos no seleccionados & 0 & .0 \\
\hline Total & 107 & 100.0 \\
\hline $\begin{array}{r}\text { a. Si está activada la ponderación, consulte la Tabla de clasificación para ver el número total de casos. } \\
\text { Fuente: elaboración propia con resultados de la investigación. }\end{array}$
\end{tabular}


En la Tabla 3 se presenta la verosimilitud del modelo, el estadístico -2LL mide hasta qué punto se ajusta bien de los datos, cuanto más pequeño sea el valor, será mejor el ajuste. $L a R$ cuadrado de Cox y Snell es un coeficiente de determinación generalizado, con un valor de 0.344 que indica que un $34,4 \%$ de la variación de la variable dependiente es explicada por las características de la administración. La $R$ cuadrado de Negelkerke, es una versión corregida de la R cuadrada de Cox y Snell, en este caso con un valor de 0.461 que equivale a $46,1 \%$.

La Tabla 4 de clasificación es muy parecida a la empleada para valorar una prueba diagnóstica, es la que permite evaluar el ajuste del modelo de regresión logística. En este paso el modelo ha clasificado correctamente a un 55,7\% de todos los casos.
La Tabla 5, variables en la ecuación, muestra en la columna B los valores de los coeficientes del modelo de regresión logística binaria, mismos que se usan para la función de regresión tal como se aprecia en la Tabla 6. La columna E.T. corresponde a los errores estándar de los coeficientes. La columna de Wald corresponde al estadístico de Wald que testea la hipótesis de si los coeficientes son iguales a «0» y que sigue una distribución $X^{2}$ con un nivel de significancia que son los que aparecen en la columna «Sig». El «Exp(B)» es el incremento o disminución que se produce en el cociente $\operatorname{pr}\left\{s_{i}\right\} / \operatorname{pr}\{$ no $\}$ pertenecen al grupo de empresas con rendimientos superiores al $20 \%$. En la Tabla 6, variables en la ecuación, se observan las características que se incluyeron en la función logística binaria, que permite clasificar a las empresas con rendimientos superiores a $20 \%$.

Tabla 3. Resumen del Modelo

Table 3. Model data

\begin{tabular}{c|c|c|c}
\hline Paso & $\begin{array}{c}-2 \text { Log de la } \\
\text { Verosimilitud }\end{array}$ & $\begin{array}{c}\text { R cuadro de Cox } \\
\text { y Snell }\end{array}$ & $\begin{array}{c}\text { R cuadro de } \\
\text { Nagelkerke }\end{array}$ \\
\hline 4 & $100.818^{c}$ & 0.344 & 0.461 \\
\hline
\end{tabular}

Fuente: elaboración propia con resultados de la investigación.

Tabla 4. Tabla de clasificación a,b

Table 4. Classification a,b

\begin{tabular}{|c|c|c|c|c|c|}
\hline & \multirow{3}{*}{ Observado } & & \multicolumn{3}{|c|}{ Pronosticado } \\
\hline & & & \multicolumn{2}{|c|}{ Rendimiento } & \multirow{2}{*}{$\begin{array}{c}\text { Porcentaje } \\
\text { correcto }\end{array}$} \\
\hline & & & .00 & 1.00 & \\
\hline \multirow[t]{3}{*}{ Paso 0} & \multirow{2}{*}{ Rendimiento } & .00 & 59 & 0 & 100.0 \\
\hline & & 1.00 & 47 & 0 & 0.0 \\
\hline & \multicolumn{2}{|c|}{ Porcentaje global } & & & 55.7 \\
\hline
\end{tabular}

a. En el modelo se incluye una constante.

b. El valor de corte es .500

Fuente: elaboración propia con resultados de la investigación. 
Tabla 5. Variables en ecuación

Table 5. Variables in the equation

\begin{tabular}{|c|c|c|c|c|c|c|c|}
\hline & & $B$ & E.T. & Wald & gl & Sig. & $\operatorname{Exp}(B)$ \\
\hline \multirow[t]{2}{*}{ Paso $1^{a}$} & Rubro Afectado & .630 & .156 & 16.322 & 1 & .000 & 1.877 \\
\hline & Constante & -1.057 & .295 & 12.819 & 1 & .000 & .348 \\
\hline \multirow[t]{3}{*}{ Paso $2^{b}$} & $\begin{array}{l}\text { Financiamiento Moneda } \\
\text { Extranjera }\end{array}$ & 1.647 & .616 & 7.141 & 1 & .008 & 5.190 \\
\hline & RubroAfectado & .515 & .165 & 9.775 & 1 & .002 & 1.674 \\
\hline & Constante & -1.252 & .318 & 15.457 & 1 & .000 & .286 \\
\hline \multirow[t]{4}{*}{ Paso $3^{c}$} & $\begin{array}{l}\text { Financiamiento Moneda } \\
\text { Extranjera }\end{array}$ & 1.398 & .629 & 4.947 & 1 & .026 & 4.048 \\
\hline & Rubro Afectado & .478 & .169 & 8.037 & 1 & .005 & 1.614 \\
\hline & $\begin{array}{l}\text { Instrumento Cobertura } \\
\text { Deudas }\end{array}$ & 1.995 & 1.008 & 3.913 & 1 & .048 & 7.350 \\
\hline & Constante & -1.378 & .333 & 17.079 & 1 & .000 & .252 \\
\hline \multirow[t]{5}{*}{ Paso $4^{d}$} & $\begin{array}{l}\text { Financiamiento Moneda } \\
\text { Extranjera }\end{array}$ & 1.938 & .864 & 5.029 & 1 & .025 & 6.946 \\
\hline & Rubro Afectado & .627 & .186 & 11.355 & 1 & .001 & 1.872 \\
\hline & Instrumento Cobertura & -1.011 & .498 & 4.116 & 1 & .042 & .364 \\
\hline & $\begin{array}{l}\text { Instrumento Cobertura } \\
\text { Deudas }\end{array}$ & 3.505 & 1.448 & 5.855 & 1 & .016 & 33.275 \\
\hline & Constante & -1.441 & .342 & 17.785 & 1 & .000 & .237 \\
\hline
\end{tabular}

a. Variable(s) introducida(s) en el paso 1: Rubro Afectado.

b. Variable(s) introducida(s) en el paso 2: Financiamiento Moneda Extranjera.

c. Variable(s) introducida(s) en el paso 3: Instrumento Cobertura Deudas.

d. Variable(s) introducida(s) en el paso 4: Instrumento Cobertura.

Fuente: elaboración propia con resultados de la investigación.

Tabla 6. Ecuación de regresión logística

Table 6. Logistic regression equation

$$
\begin{aligned}
& \text { Ecuación } \\
& \qquad \begin{array}{l}
\mathrm{P}\{\mathrm{Y}=1\}=\frac{e^{Z}}{1+e^{Z}} \\
Z=-1.441+1.938 X_{1}+0.627 X_{2}-1.011 X_{3}+3.505 X_{4}
\end{array}
\end{aligned}
$$

Donde:

\begin{tabular}{c|l}
\hline Variable & \multicolumn{1}{c}{ Interpretación } \\
\hline $\mathrm{P}$ & $\begin{array}{l}\text { Probabilidad en función a los valores de las razones financieras y la función de regresión } \\
\text { logística binaria }\end{array}$ \\
\hline $\mathrm{Z}$ & Puntuación discriminante $\mathrm{Z}$ \\
\hline $\mathrm{E}$ & 2.71828 \\
\hline$X_{1}$ & Cantidad de financiamiento en moneda extranjera del total de las deudas \\
\hline$X_{2}$ & Rubro que más ha afectado las variaciones de tipo de cambio \\
\hline$X_{3}$ & $\begin{array}{l}\text { Utilización de instrumentos o estrategias de cobertura para los ingresos en divisa extranjera } \\
\text { para evitar los efectos adversos de una posible baja de precio de la divisa extranjera }\end{array}$ \\
\hline$X_{4}$ & $\begin{array}{l}\text { Utilización de instrumentos o estrategias de cobertura para deuda para evitar los efectos } \\
\text { adversos de una posible alza de precio de la divisa extranjera }\end{array}$ \\
\hline
\end{tabular}

\footnotetext{
Fuente: elaboración propia con resultados de la investigación.
} 
La ecuación de regresión logística se usa para predecir la probabilidad de pertenecer al grupo de las empresas Rentables $=1$ o No Rentables $=$ 0 , de una empresa en función al valor de las características predominantes en su administración de riesgos cambiarios en ambientes de contingencia. Si una empresa tiene un valor de menos de 0.50 al sustituir los valores de sus valores medios en la ecuación de regresión logística, se clasificaría como No rentable; por el contrario, si el resultado fuera mayor a 0.50 , se clasificaría como Rentable, donde se consideran empresas Rentables las que tienen márgenes de rentabilidad superiores al $20 \%$ y no rentables a aquellas con rendimientos entre 0 y $20 \%$.

Los resultados del análisis estadístico permiten rechazar la hipótesis nula y aceptar la alternativa planteadas debido a que de los 23 factores que se evaluaron solo cuatro (son las variables que resultaron ser estadísticamente significativas en la RL) logran que las empresas obtengan rendimientos superiores al $20 \%$, siendo: (1) son la cantidad de financiamiento en moneda extranjera, (2) rubro que más ha afectado el tipo de cambio, el cual propende a cero, lo que significa que prácticamente no tiene afectaciones, (3) la utilización de instrumentos financieros derivados de cobertura para los ingresos en divisa extranjera y (4) la utilización de instrumentos financieros derivados de cobertura para la deuda en divisa extranjera.

\section{CONCLUSIONES}

Se desarrolló un análisis estadístico de regresión logística binaria para determinar las características de la administración prevalecientes de las empresas de la ciudad de Oaxaca y la Ciudad de México con operaciones internacionales, ya sea que exporten y/o importen, que clasificó a las compañías en dos grupos: (1) con rendimientos de 0 a $20 \%$, (2) las que tienen rendimientos mayores del $20 \%$ y considerados como rentables.

En el análisis estadístico se incluyeron variables sobre el perfil de los administradores como fue la escolaridad, su edad, el máximo grado de estudios, opinión sobre el riesgo de negocio y la existencia de personal calificado para el manejo de instrumentos financieros derivados, el uso o carencia de asesoría para realizar las operaciones con el exterior; en lo referente a las operaciones que las compañías se consideró la cantidad de financiamiento extranjero, los ingresos en moneda extranjera, utilización de instrumentos financieros derivados para cubrir los riesgos de los ingresos y las deudas en moneda extranjera y su frecuencia de uso.

El resultado del análisis de regresión logística encontró que sólo cuatro variables de las 23 evaluadas fueron estadísticamente significativas para determinar que las empresas logren obtener rendimientos superiores al $20 \%$, por lo cual se puede decir que aquellas empresas que usan financiamiento en moneda extranjera, las que identifican los rubros que más afectan las variaciones cambiarias y para ello utilizan instrumentos financieros derivados para mantener el valor de sus ventas en moneda extranjera, es el caso de usar un contrato corto de dólares para asegurar el precio de venta de los dólares que perciben por los ingresos de sus ventas al extranjero, así como el uso de coberturas para fijar los precios máximos que pagarán por la compra de dólares en el caso que suban de precio al momento de saldar sus deudas.

Los resultados de esta investigación demuestran que las compañías estudiadas para enfrentar la incertidumbre de su entorno identifican las variables que producen mayor impacto en sus resultados financieros, en este caso la variabilidad del tipo de cambio, en ese sentido coinciden con lo que postula la teoría de la contingencia y las investigaciones de 
Burns Y Stalker (1961), Lawrence y Lorsch (1967), y por ello emprenden nuevas estrategias como es el uso de los instrumentos financieros derivados y coinciden con Chandler (1990), quien resalta que las compañías acogen nuevas estrategias para adaptarse al medio ambiente. Aunque las características del administrador financiero no resultaron ser relevantes contrario a los hallazgos de Dolde (1993) quien encontró evidencia de que más del $45 \%$ de las empresas estadounidenses tenían un profesional a tiempo completo dedicado a la gestión del riesgo.

El grupo de empresas con rendimientos superiores al $20 \%$ analizadas de este documento se caracterizaron por usar instrumentos financieros derivados como cobertura, lo cual coincide con las investigaciones de Sierra y Londoño (2010) quienes también encontraron el uso de estos instrumentos en las empresas colombianas para la administración del riesgo, considerando que además es otro país latinoamericano que realiza esta práctica, porque en los países desarrollados Jorion (1990) encontró que el nivel de cobertura guarda una relación directa con la proporción de ventas, entonces pareciera ser que las ventas extranjeras de las empresas que realizan operaciones internacionales dependen más del nivel de ventas principalmente.

Las evidencias encontradas muestran la administración del riesgo cambiario en ambientes de contingencia de las empresas exportadoras y/o importadoras de Oaxaca y Ciudad de México se caracterizan principalmente por prevalecer el uso de instrumentos financieros derivados para las compañías mayormente rentables.

\section{REFERENCIAS}

Arango, E., \& Arroyave, J. (2011). Swaps de tasa de interés y de cruce de monedas como herramientas de cobertura para las empresas colombianas. Revista EIA, 16(8), 189-205.

Burns, T., \& G.M. Stalker. (1961). The Management of Innovation. Recuperado de http://www.oxfordscholarship.com/view /10.1093/acprof:oso/9780198288787.00 1.0001/acprof-9780198288787

Cadogan, J. W., Diamantopoulos, A., \& De Mortanges, C. P. (1999). A measure of export market orientation: Scale development and cross-cultural validation. Journal of International Business Studies, 30(4), 689-707.

Chandler, A. D. (1990). Estrategia y estructura: los capítulos en la historia de la empresa industrial. Cambridge: MIT Press.

Chell, E. (1991). The entrepreneurial personality: Concepts, Cases and Catagories. Routledge Small Business Series. London: Routledge.

Chiavenato, I. (2006). Introducción a la teoría general de la administración. México: Mc Graw Hill

Chowdhury, S., \& Endres, M. (2005). Gender difference and the formation of entrepreneurial self-efficacy. Presentado en The United States Association of Small Business (USASBE) Annual Conference, Indian Wells, CA.

Clavijo, W., \& Roberto, K. (2009). Las operaciones de cobertura y sus efectos fiscales. Revista Impuestos, 153, 22-25. 
Cooper, A. C., Gimeno-Gascon, F.J., \& Woo, C.Y. (1994). Initial human and financial capital as predictors of new venture performance. Journal of Business Venturing, 9(5), 371-395.

Damico, A. (2011). El convenio de pagos y créditos recíprocos de ALADI como una herramienta de facilitación del comercio ante la crisis internacional. Revista De Derecho (15105172), 10(20), 185-225.

Dolde, W. (1993). Use of Foreign Exchange and Interest Rate Risk Management in Large Firms. Working Paper, University of Connecticut, Storrs, CT.

Entrialgo, M. (2000). Habilidades adquiridas y función empresarial. Iniciativa emprendedora y empresa familiar, 20 (enero-febrero), 21-29.

Fernández, E., \& Junquera, B. (2001). Factores determinantes en la creación de empresas: una revisión de la literatura. Papeles de Economía Española, (89-90), 322-342.

Follet, M. (1960). Administración dinámica. México: Herrero Hermanos.

García, F., \& Avella, L. (2007). Intensidad exportadora y percepción de barreras a la exportación: un estudio de casos. Investigaciones Europeas de Dirección y Economía de la Empresa, 13(3), 93-106.

Gray, S., \& Place, J. (2003). Derivados financieros. México: Centro de Estudios Monetarios Latinoamericanos.

Gupta, V. K., \& York, A. S. (2008). The effects of geography and age on women's attitudes towards entrepreneurship. Evidence from the state of Nebraska. The International
Journal of Entrepreneurship and Innovation, 9(4), 251-262.

Hatch, M. J. (1997). Organization Theory: Modern, Symbolic and Postmodern Perspectives. New York: Oxford.

Hair, J., Anderson R., Tatham R., \& Black, W. (1999). Análisis multivariante. México: Pearson Prentice Hall.

INEGI (2017). Información oportuna sobre la balanza comercial de mercancías de México. Recuperado de http://www.beta.inegi.org.mx/app/salad eprensa/noticia.html?id=3285

Jorion, P. (1990). The Exchange Rate Exposure of U.S. Multinationals. Journal of Business, 13(11), 375-386. Recuperado de https://www.jstor.org/stable/2353153?s eq=1\#page_scan_tab_contents

Kauffman, S. (2000). Estrategia mente global. Recuperado de https://www.uv.mx/iiesca/files/2013/01/ estrategia1999-2000.pdf

Kaynak, E., \& Kothari, V. (1984). Export behaviour of small and medium-sized manufacturers: some policy guidelines for international marketers. Management International Review, 24(2), 61-69.

Kedia, S., \& Mozumdar, A. (2003). Foreign currency-denominated debt: An empirical examination. Journal of Business, 76(4), 521-546.

Kohli, A. K., \& Jaworski, B. J. (1993). Market Orientation: Antecedents and Consequences. Journal of Marketing, 57(3), 53-70.

Kong, V. X., \& Zhang, J. (2010). The effect of managerial education and firm- 
ownership structure empirical evidence from Chinese listed firms. The Chinese Economy, 43(6), 34.53.

Koontz, H., \& Weihrich, H. (2012). Administración: una perspectiva global y empresarial. (14a. ed.). Mc Graw Hill.

Lawrence, P. R., \& Lorsch, J. W. (1967). Differentiation and integration in complex organizations, Administrative Science Quarterly, 12(1), 1-47. Recuperado de https://www.jstor.org/stable/2391211?s eq=1\#page_scan_tab_contents

Levi, M. (2006). Finanzas Internacionales. (3a ed.). México: Mc Graw Hill.

Lewis, P. (2006). The quest for invisibility: female entrepreneurs and the masculine norm of entrepreneurship. Gender, Work and Organization, 13(5), 453-469.

López, G. (2010). Finanzas Corporativas: un enfoque latinoamericano. ( 2 a $\quad$ ed.). Argentina: Alfaomega.

Madura, J. (2009). Administración Financiera Internacional. (9a ed.). México: Cengage Learning.

McFarland, D. (1974). Management: Principles and Practices. (4th ed.). McMillan. New York.

McMillan, I. C., Siegel, R., \& Narasimha, P. N. (1985). Criteria used by venture capitalists to evaluate new venture proposals. Journal of Business Venturing, 1(1), 119128.

Montesinos, L., Luna, E., Hernández, S., \& Tinoco Z. M. A. (2009). Muestreo estadístico. Tamaño de muestra y estimación de parámetros. Colima, México: Universidad de Colima.
OCDE (2017). Estudios Económicos de la OCDE. México: OCDE.

Ong, J. W., Ismail, H.B., \& Goh, G. G. (2010). The competitive advantage of small and medium enterprises (smes): the role of entrepreneurship and luck. Journal of Small Business and Entrepreneurship, 23(3), 373-391.

ProMéxico (2016). Directorio de exportadores. Entidad federativa y producto. ProMéxico. Recuperado de https://bit.ly/2HMaGNp

ProMéxico y SE (2016). Hacer sinergia para competir. Redexporta: un programa para crear y consolidar redes de exportación en México.

Ross, S., Westerfield, R., \& Jaffe, J. (2005). Finanzas Corporativas. (7a. ed.). México: McGraw Hill/Interamericana de México.

Sierra, J. \& Londoño, D. (2010). Cobertura con derivados en empresas manufactureras colombianas: análisis previo a la apertura del mercado de derivados en la bolsa de valores de Colombia. Cuad. Adm., 23(41), 237-260. Recuperado de http://www.scielo.org.co/pdf/cadm/v23n 41/v23n41a11.pdf

Secretaría de Información Empresarial Mexicano (SIEM). (2016). Estadisticas de empresas que exportan, importan por Estado. Recuperado de https://www.siem.gob.mx/siem/portal/e stadisticas/Est_Explmp_xEdo.asp

Storey, D. J. (1994). Understanding the small business sector. London, UK: Routledge. 
Ucbasaran, D., Westhead, P., \& Wright, M. (2008). Opportunity identification and pursuit: does an entrepreneur's human capital matter. Small Business Economics, 30(2), 153-173.

Veciana, J. M. (1989). Características del empresario en España. Cuadernos de Economía, 39, 19-36.
Zapata, E. (2005). Las pymes y su problemática empresarial: análisis de casos. En G. Calderón \& G. Castaño. Investigación en administración en América Latina: Evolución y resultados. Manizales, Colombia: Universidad Nacional de Colombia. 